\title{
Study of the beam path distortion profiles generated by a two-axis tilt single-mirror laser scanner
}

\author{
Moustapha Hafez \\ Service de Robotique et Systèmes \\ Interactifs \\ Commissariat à l'Energie Atomique \\ Fontenay Aux Roses, France \\ E-mail: moustapha.hafez@cea.fr \\ Thomas Sidler, MEMBER SPIE \\ René-Paul Salathé \\ Swiss Federal Institute of Technology \\ Institute of Applied Optics \\ Department of Micro-Engineering \\ $\mathrm{CH}-1015$ Lausanne, Switzerland
}

\begin{abstract}
Beam distortion profiles are studied for scanning devices that have a single mirror with two rotational degrees of freedom (DOF), also named tip/tilt scanners. The case of a fast steering scanner used for high power material processing applications is studied. The scanner has a bandwidth of $700 \mathrm{~Hz}$, a range of motion of $\pm 52 \mathrm{mrad}( \pm 3 \mathrm{deg})$, and a resolution $<5 \mu \mathrm{rad}$. The main dominant parameters that affect the distortion profile are identified. Furthermore, a vector analysis is derived to represent these distortions, and equations of the correction factors used to compensate for the systematic errors are proposed. A final accuracy better than $0.05 \%$ is obtained when these compensation factors are taken into account. The derivation proposed here can be extended to any scanning device with a single mirror with two-rotation DOF. () 2003 Society of Photo-Optical Instrumentation Engineers. [DOI: 10.1117/1.1557694]
\end{abstract}

Subject terms: tip/tilt scanner; beam path distortions; laser material processing.

Paper 020038 received Feb. 4, 2002; revised manuscript received Sep. 3, 2002; accepted for publication Sep. 26, 2002.

\section{Introduction}

Laser scanners are used in a variety of optical systems and steer different types of lasers for various applications such as imaging, industrial material processing, biomedicine, ophthalmology, and intersatellite communications. Image distortions are caused by various factors. To design and use opto-mechanical systems with very high accuracy, sources of error must be accurately identified. They should then be quantified and calculated so that they can be rectified. Beam path distortion (BPD), which is completely independent from lens aberrations, may come from either the scan head or the imaging system. It is caused by path length variations for different portions of the beam. Portions of the image may be blurred or may focus before or after the image plane. Alternatively, portions of the image beam may be directed to an incorrect position on the image plane.

The common configuration for deflecting laser beams in two dimensions is based on two single-axis galvanometric scanners placed perpendicular to each other. In recent years, these fast scanners have proved to be both very reliable and accurate. They are widely available in the marketplace and have wide-ranging technical variations. However, such a configuration is rather bulky and cannot satisfy the needs of an industry that is looking for compact solutions for an easy integration of these scanning heads into their production lines. In addition, two separate mirrors lead to optical aberrations when these scanning heads are used in conjunction with scanning lenses, which are specially designed for a single entrance pupil. The distortions and correction factors for such scanning configurations have been previously derived. ${ }^{1,2}$

A compact fast-steering tip/tilt laser scanner with a single mirror could act as a key component in diverse applications as it provides a much easier, simpler, and much more compact way to perform a 2-D scan. Previous work presented different two-axis tilt mechanisms with one single mirror. ${ }^{3-5}$ We introduce a highly innovative singlemirror tip/tilt laser scanner and focuses on the BPD resulting from such scanner, which was designed for material processing applications. A single mirror with two rotational DOF generates distortions that are different from the pincushion distortion resulting from the use of two separate mirrors. ${ }^{1,2}$ Based on a vector analysis of the beam path, the distortion profiles are derived for such a scanner. This vector derivation can be compared to the holographic techniques applied to deformation and shape measurements. ${ }^{6,7}$ The main dominant parameters that affect the distortion profile are identified, and equations of the correction factors used to compensate for the systematic errors are proposed.

\section{Scanner Description}

The system studied in this work (see Fig. 1) requires a multidisciplinary approach of the optics, mechanics, electronics, sensor, and control fields. The main components are the mirror, the motors, the feedback sensor that determines the mirror position around the two axes of tilt, and the control system that connects the feedback sensor to the motors. Electromagnetic actuators are used to drive the mirror. They are based on magnets and coils. Magnets are glued directly on the back of the mirror, whereas coils are fixed on the stationary part. The guiding system is based on a cone-ball bearing, which fixes the translation DOF and releases the rotation DOF. It is a standard and cheap construction, which simplifies the overall design of the scanner. A preload magnetic force is used to hold the moving part. This force also creates sufficient rotation stiffness around the mirror axis. However, this force should not be too high in order to minimize friction in the bearing. The position 


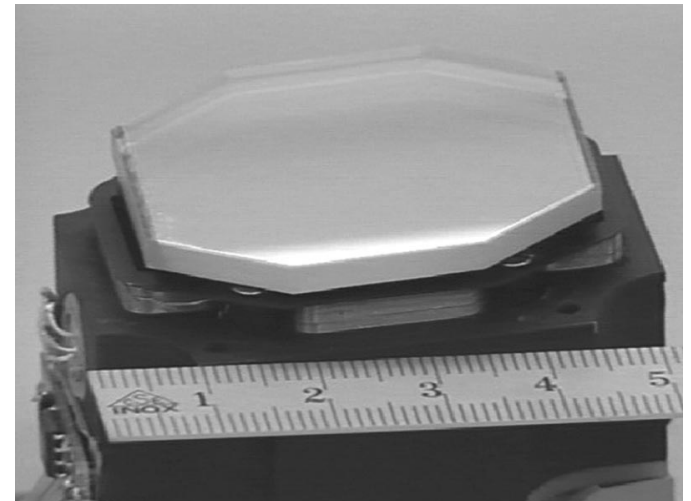

Fig. 1 Compact tip/tilt laser scanner with a large reflecting mirror.

transducer is placed at the back of the mirror. A laser pen is used to point on the back of the mirror and then on a 2-D position sensitive detector (PSD). The design, simulations, and experimental investigations of this scanner have been previously published. ${ }^{8}$ Table 1 indicates the specifications and performance of the scanner.

\subsection{Scanner Application for MicroSpot Welding}

The compact scanner represents a key module in aim-andshoot applications due to its high dynamic performance and accuracy. The scanner has been mounted on a compact multisensor real-time monitoring optical head (see Fig. 2) to achieve microspot welding. ${ }^{9}$ One of the sensors (S1) is used to determine temperature evolution during the spot welding process. It is composed of two photodiodes, a silicon photodiode (S1A) and a germanium photodiode (S1B). Another sensor (S2) is used to detect plasma emission. A third sensor (S3) is used to monitor back-reflected light, which gives information about the exact time at which the melting process occurs, and the fourth sensor (S4) is used to monitor the optical fiber output power. A vision channel and a CCD camera are used to control the position of the laser spot on the parts to be processed. This whole system will be soon available on the market and produced by the Swiss laser manufacturer Lasag.

Table 1 Tip/tilt scanner specifications.

\begin{tabular}{lc}
\hline \hline Parameter & Performance \\
\hline Number of axes & 2 (Tip-tilt) \\
Range of motion & $+52 \mathrm{mrad}( \pm 3 \mathrm{deg})$ \\
Repeatability & $<50 \mu \mathrm{rad}$ \\
Resolution & $<5 \mu \mathrm{rad}$ \\
Settling time for & $<10 \mathrm{~ms}$ \\
maximum deflection & $700 \mathrm{~Hz}$ \\
Bandwidth & $18 \mathrm{rad} / \mathrm{s}$ \\
Angular velocity & $30 \times 40 \mathrm{~mm}$ \\
Mirror active area & $50 \times 40 \times 30 \mathrm{~mm}$ \\
Size of the scanner & $90 \mathrm{~g}$ \\
Weight & $\pm 10 \mathrm{~V}$ \\
Input voltage range & \\
\hline \hline
\end{tabular}

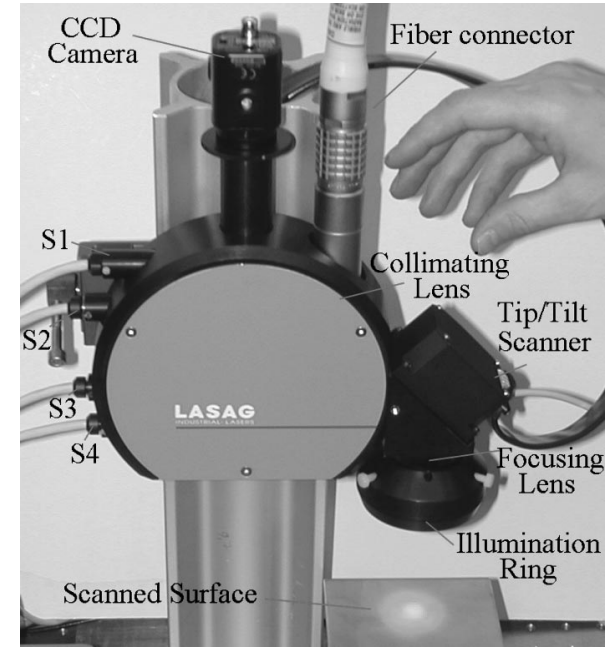

Fig. 2 Compact multisensor laser scanning head. S1 is the temperature sensor composed of two photodiodes ( $\mathrm{Si}$ and $\mathrm{Ge}$ ) in a sandwich configuration. S2 is the plume sensor, S3 detects the back-reflected light of Nd:YAG on the irradiated part, and S4 is the monitoring sensor for the laser to detect pulse shape.

\section{Beam Path Distortion for Tip/Tilt Scanners}

When a laser beam is reflected by a mirror, which is tilted around the two orthogonal axes in a plane parallel to the mirror, onto a second surface (target plane), geometrical distortions result, as illustrated in Fig. 3. Such distortions are defined as "fan-shaped" distortions in this study. These distortions depend on several factors: the angle of incidence of the laser beam at the mirror's reflecting surface $\gamma$, where $(\beta=90 \mathrm{deg}-\gamma)$, the tip and tilt angles of the mirror $\alpha_{x}$ and $\alpha_{y}$, the working distance $d$ that separates the target surface from the mirror's center of rotation, the orientation of the target plane, and the distance $D$ between the center of rotation and the center of reflection.

\subsection{Vector Analysis}

A vector analysis is introduced to determine and relate the actual position of the spot on the target plane to the tip and tilt angles $\alpha_{x}$ and $\alpha_{y}$ of the mirror. Figure 4 shows a laser

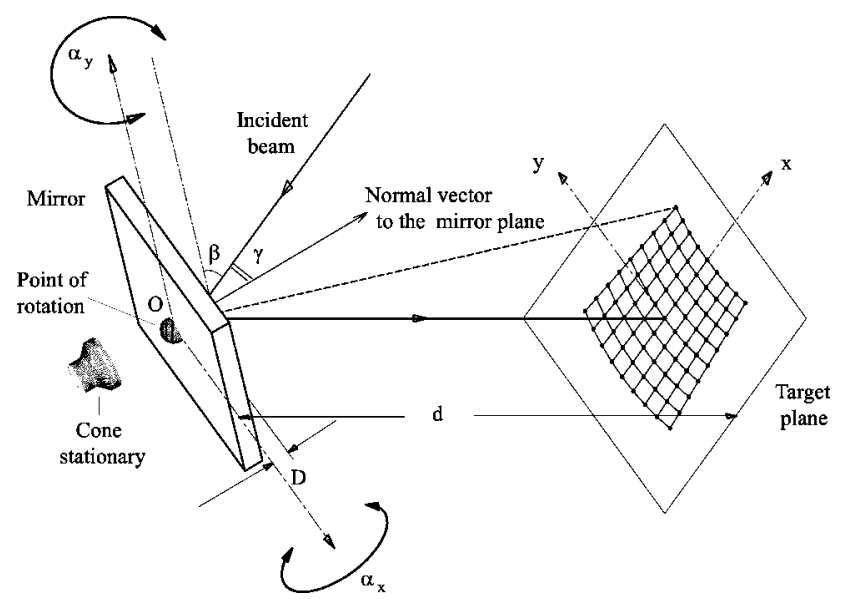

Fig. 3 Fan-shaped distortion on the working surface resulting from a single mirror system, which can tilt in two-rotational DOF. 


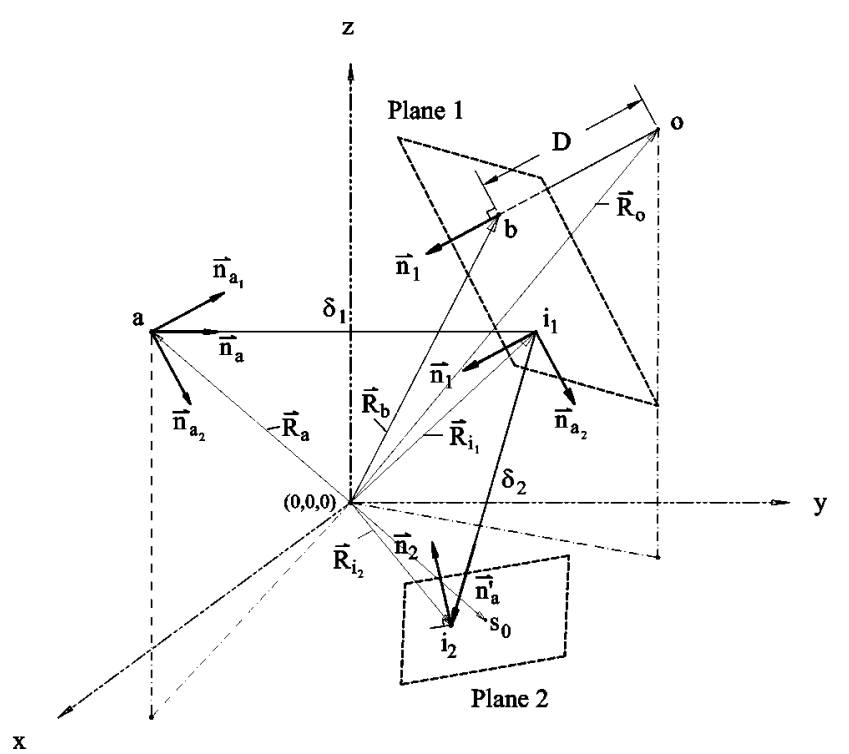

Fig. 4 Vector analysis to determine the points of impact between a laser beam and two planes positioned randomly in space. In this case, plane 1 is the mirror plane and plane 2 is the target plane.

beam that has a first point of impact $i_{1}$ with the mirror plane (plane 1), and a second point of impact $i_{2}$ with the target plane (plane 2). Point $a$ is defined as the incident point of the laser beam, and point $o$ is the point of rotation of the mirror. Point $b$ is a point on the mirror plane and is located on the normal vector to the mirror plane passing through $o$.

The vector to the point of impact $i_{1}$ with the mirror plane is given by:

$\vec{R}_{i_{1}}=\vec{R}_{a}+\delta_{1} \cdot \vec{n}_{a}$,

where $\vec{n}_{a}$ is the unit vector in the direction of the laser beam, $\delta_{1}$ is a scalar, and $\vec{R}_{a}$ is the vector from the origin $(0,0,0)$ to point $a$.

For any plane in space, the scalar product of the normal vector to the plane by any vector that starts from the origin to a point located on the surface of the plane is a constant $K$. Therefore, for the mirror plane, where $\vec{n}_{1}$ is the normal vector to the plane and both points $b$ and $i_{1}$ lie on the plane, the previous definition applies:

$\vec{n}_{1} \cdot \vec{R}_{i_{1}}=\vec{n}_{1} \cdot \vec{R}_{b}=K \Rightarrow \vec{n}_{1} \cdot\left(\vec{R}_{i_{1}}-\vec{R}_{b}\right)=0$,

and

$\vec{R}_{b}=\vec{R}_{o}+D \cdot \vec{n}_{1}$.

Based on the previous equations, the scalar $\delta_{1}$ can be written as:

$\delta_{1}=\frac{\vec{n}_{1} \cdot\left(\vec{R}_{o}-\vec{R}_{a}\right)+D}{\left(\vec{n}_{1} \cdot \vec{n}_{a}\right)}$.

Once the distance $\delta_{1}$ is determined, the point of impact $i_{1}$ is calculated from Eq. (1). The second point of impact $i_{2}$ with the target plane is determined in the same way.

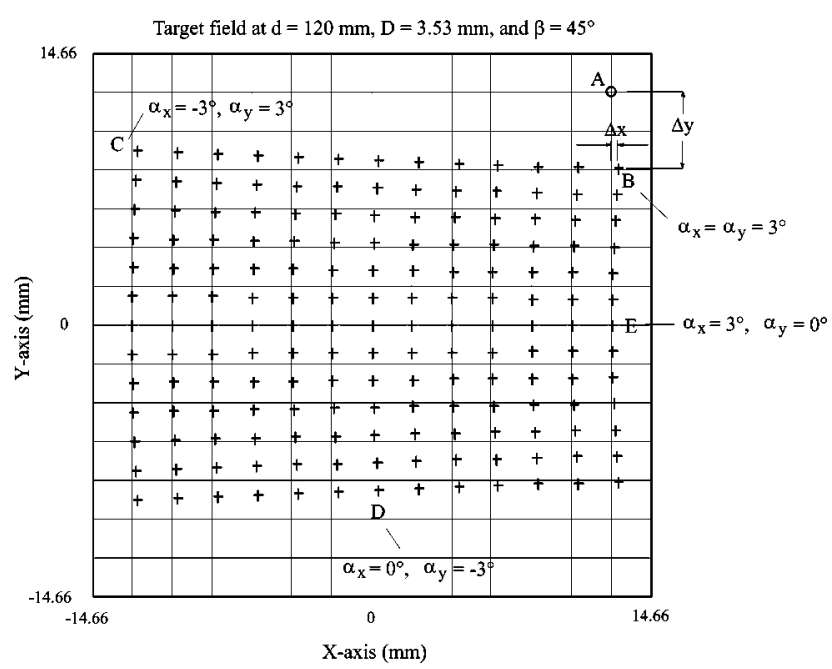

Fig. $5 X Y$ distortions on a target plane positioned $120 \mathrm{~mm}$ from the center of rotation of a mirror, which tilts by $\left(\alpha_{x}=\alpha_{y}= \pm 3 \mathrm{deg}\right)$ and where $\beta=45$ deg.

$\vec{R}_{i_{2}}=\vec{R}_{i_{1}}+\delta_{2} \cdot \vec{n}_{a}^{\prime}$,

where $\vec{n}_{a}^{\prime}$ is the unit vector along the reflected beam. The vectors $\vec{n}_{a}, \vec{n}_{a}^{\prime}$, and $\vec{n}_{1}$ form a third plane (according to the law of reflection). Therefore $\vec{n}_{a}^{\prime}$ can be represented in terms of $\vec{n}_{1}$ and $\vec{n}_{a}$. First, $\vec{n}_{a}$ is decomposed into two components: $\vec{n}_{a_{1}}$ parallel to $\vec{n}_{1}$, and $\vec{n}_{a_{2}}$ orthogonal to $\vec{n}_{1}$.

$\vec{n}_{a}=\vec{n}_{a_{1}}+\vec{n}_{a_{2}}$.

The reflection on the mirror plane inverts $\vec{n}_{a_{1}}$ and keeps $\vec{n}_{a_{2}}$ in the same direction. The resulting unit vector in the new direction $\vec{n}_{a}^{\prime}$ then is equal to:

$\vec{n}_{a}^{\prime}=-\vec{n}_{a_{1}}+\vec{n}_{a_{2}} \Rightarrow \vec{n}_{a_{2}}=\vec{n}_{a}^{\prime}+\vec{n}_{a_{1}}$.

$\vec{n}_{a_{1}}$ can also be described in terms of the following expression:

$\vec{n}_{a_{1}}=\left(\vec{n}_{a} \cdot \vec{n}_{1}\right) \cdot \vec{n}_{1}$.

Therefore $\vec{n}_{a}^{\prime}$ is represented in terms of $\vec{n}_{1}$ and $\vec{n}_{a}$ using Eqs. (6), (7), and (8):

$\vec{n}_{a}^{\prime}=\vec{n}_{a}-2 \cdot\left(\vec{n}_{a} \cdot \vec{n}_{1}\right) \cdot \vec{n}_{1}$.

Knowing that $i_{2}$ and $s_{0}$ are two random points on the target plane, the scalar $\delta_{2}$ is described using the previous reasoning:

$\delta_{2}=\frac{\vec{n}_{2} \cdot\left(\vec{R}_{s_{o}}-\vec{R}_{i_{1}}\right)}{\left(\vec{n}_{2} \cdot \vec{n}_{a}^{\prime}\right)}$,

where $\vec{n}_{2}$ is the unit vector normal to the target plane, and $\vec{R}_{i_{1}}$ and $\vec{R}_{s_{0}}$ are the vectors from the origin $(0,0,0)$ to the points $i_{1}$ and $s_{0}$, respectively. Once $\delta_{2}$ is known, the point 


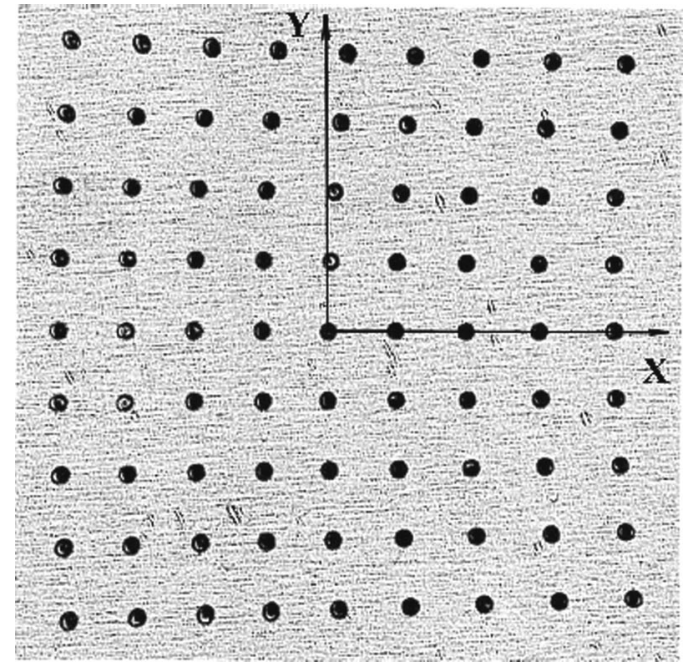

Fig. 6 Welding spots with a diameter of $400 \mu \mathrm{m}$ on a $20 \times 20 \mathrm{~mm}^{2}$ target using a single mirror scanner in a preobjective configuration. The welding spot distribution reveals the nonlinear behavior.

of impact $i_{2}$ with the second plane is determined from Eq. (5). Therefore, the previous vector analysis allows the distortions introduced in Fig. 3 to be determined.

\subsection{Distortion Profiles}

Figure 5 illustrates the fan-shaped distortion resulting from an optical configuration with a mirror positioned at $45 \mathrm{deg}$ to the incident beam, with geometrical parameters (see Fig. 3) $(d=120 \mathrm{~mm})$, and $(D=3.53 \mathrm{~mm})$, which are practical values taken from the optical head used for microspot welding previously presented in Sec. 2.1. The grid indicated in the figure corresponds to the ideal linear relation between the displacement on the target field and the scan angles $\left(X=Y=2 d \alpha_{x}=2 d \alpha_{y}\right)$. Each division of $2.094 \mathrm{~mm}$

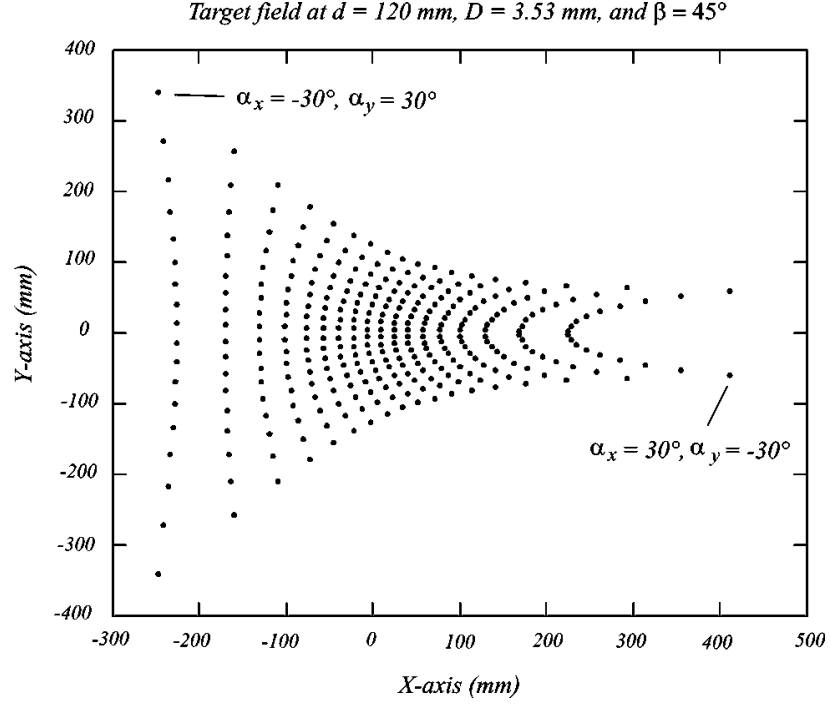

Fig. $7 X Y$ distortions on the same target for larger tilt angles $\left(\alpha_{X}\right.$ $\left.=\alpha_{y}= \pm 30 \mathrm{deg}\right)$ and where $\beta=45 \mathrm{deg}$.

corresponds to $0.5 \mathrm{deg}$. It is observed that on the $X$ axis ( $\alpha_{y}=0 \mathrm{deg}$ ), the relation between $X$ and $\alpha_{x}$ is quite linear, and the small distortions are due to the mirror surface off axis. Point $E$ on the $X$ axis, which results from $\left(\alpha_{x}\right.$ $=3 \mathrm{deg}$, and $\alpha_{y}=0 \mathrm{deg}$ ) is coinciding with the grid. On the other hand, the displacement of the spot along the $Y$ axis $\left(\alpha_{x}=0 \mathrm{deg}\right)$ is not linear but follows a curvature that seems to be constant all over the surface of the target plane shown. Point $B$ corresponds to $\left(\alpha_{x}=3 \mathrm{deg}\right.$, and $\alpha_{y}$ $=3 \mathrm{deg}$ ), whereas point $A$ corresponds to the desired linear position. It is clear that the $X$-axis error $\Delta x$ is much smaller than the $Y$-axis error $\Delta y$.

Figure 6 shows a stainless steel substrate which has been

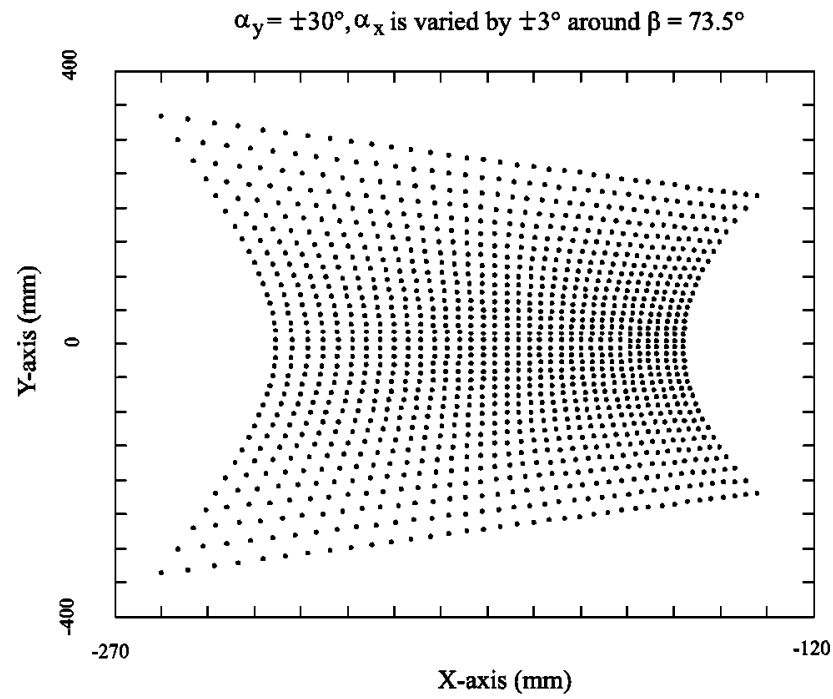

(a)

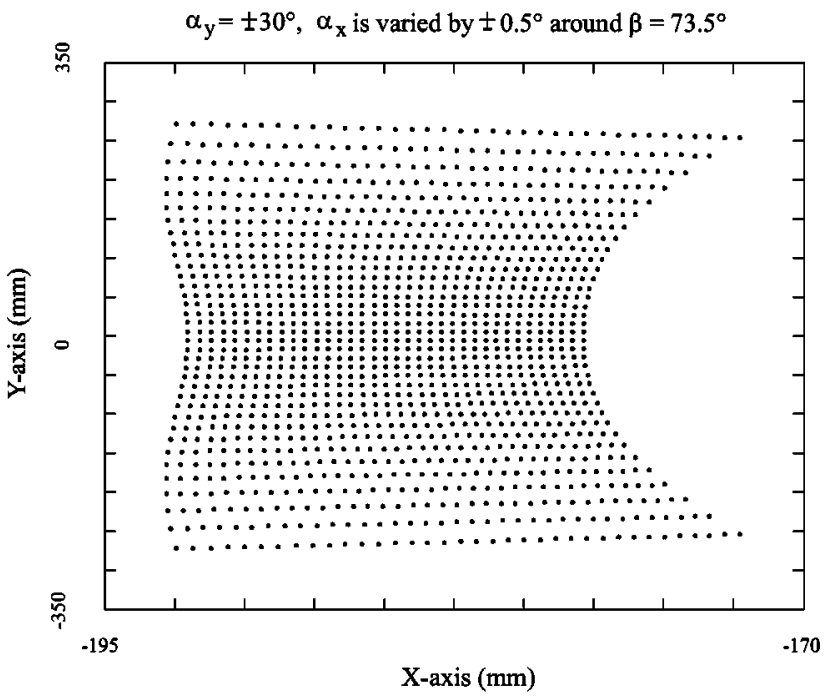

(b)

Fig. 8 (a) $X Y$ distortions on a target plane $(d=120 \mathrm{~mm})$ for tilt angles of $\alpha_{y}= \pm 30 \mathrm{deg}, \alpha_{x}$ is varied by $\pm 3 \mathrm{deg}$ around $\alpha_{X}=28.5 \mathrm{deg}$. The center of rotation is at the center of reflection, which means that $D=0$. (b) Same $X Y$ distortions as (a) but for a smaller deviation of $\alpha_{X}= \pm 0.5$ deg around $\alpha_{X}$ $=28.5 \mathrm{deg}$. 


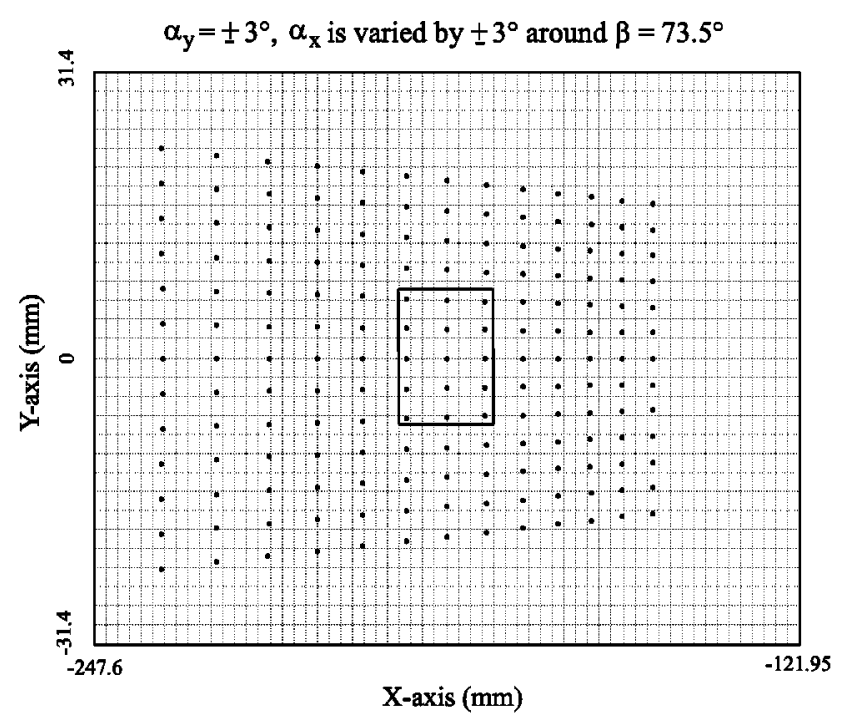

(a)

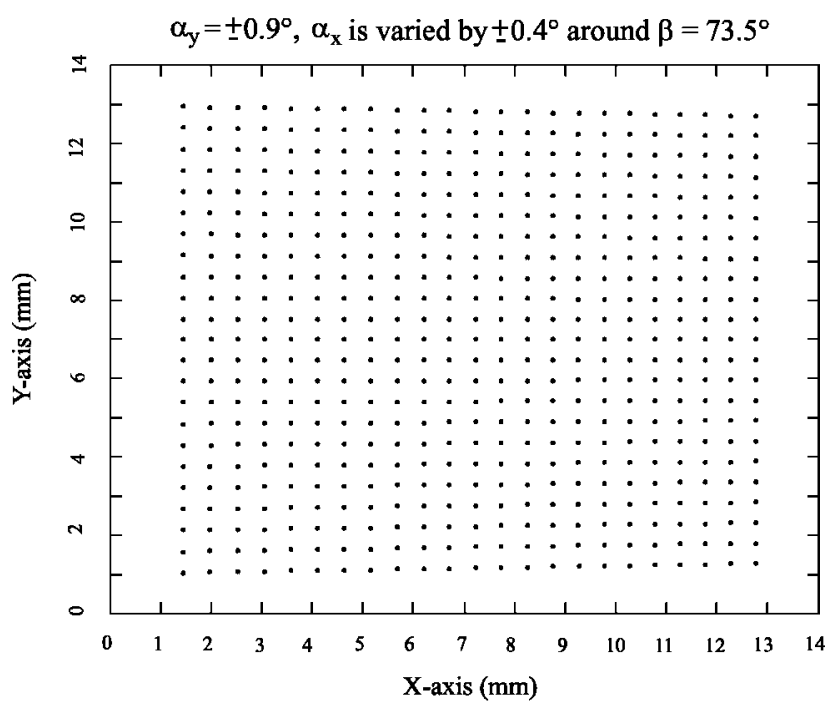

(b)

Fig. 9 (a) $X Y$ distortions for $\alpha_{y}= \pm 3$ deg and $\alpha_{x}$ is varied by \pm 3 deg around $\alpha_{x}=28.5$ deg. (b) Zoom of the area indicated in (a) $\left(\alpha_{y}= \pm 0.9 \mathrm{deg}, \alpha_{x}= \pm 0.4 \mathrm{deg}\right.$ around $\left.\alpha_{x}=28.5 \mathrm{deg}\right)$.

irradiated by a high power pulsed Nd:YAG laser with a pulse energy of $2 \mathrm{~J}$ and a pulse duration of $5 \mathrm{~ms}$. The optical head is based on a preobjective scanning configuration with an angle $\beta=45 \mathrm{deg}$, a working distance $d$ $=120 \mathrm{~mm}$, and based on a single mirror, which is tilted around a single point of rotation. The welding spots on the substrate have a diameter of $400 \mu \mathrm{m}$ all over a scan field of $20 \times 20 \mathrm{~mm}^{2}$. It should be noted that the experimental tests confirmed the vector analysis results and indicated the same nonlinear behavior as expected from Fig. 5.

Increasing the scan angles from $\alpha_{x}=\alpha_{y}= \pm 3 \mathrm{deg}$ to $\alpha_{x}=\alpha_{y}= \pm 30 \mathrm{deg}$ (10 times more than Fig. 5) gives the nonlinear distribution indicated in Fig. 7. Two interesting

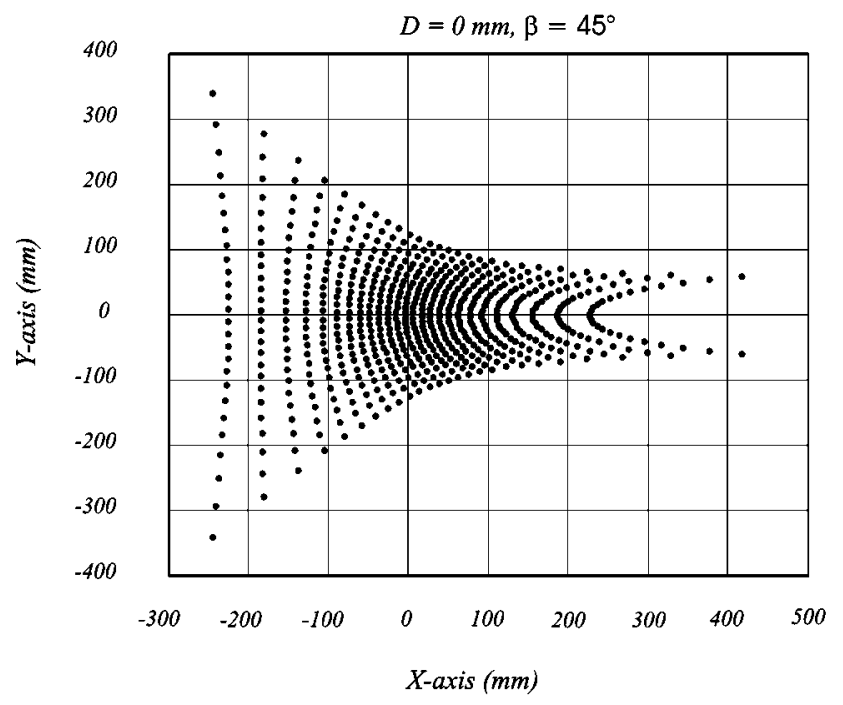

(a) points may be noticed. The first is that there is a clear symmetry around the $X$ axis all over the large scan field, which is not the case for the $Y$ axis. The second point is that the curvature behavior in the $Y$ axis gradually decreases until it reaches a specific point on the negative $X$ axis, where $\Delta x$ is zero for the different values of $\alpha_{y}$. The curvature is then inverted after this point. To get a better understanding of the point where the $X$ and $Y$ scanned lines seem to be linear and orthogonal to each other (at $X$ $=-200 \mathrm{~mm}$ ), two simulations that enlarge this area of interest are given in Figs. 8(a) and 8(b). Figure 8(a) represents the distortion profile for scan angles of $\alpha_{y}$

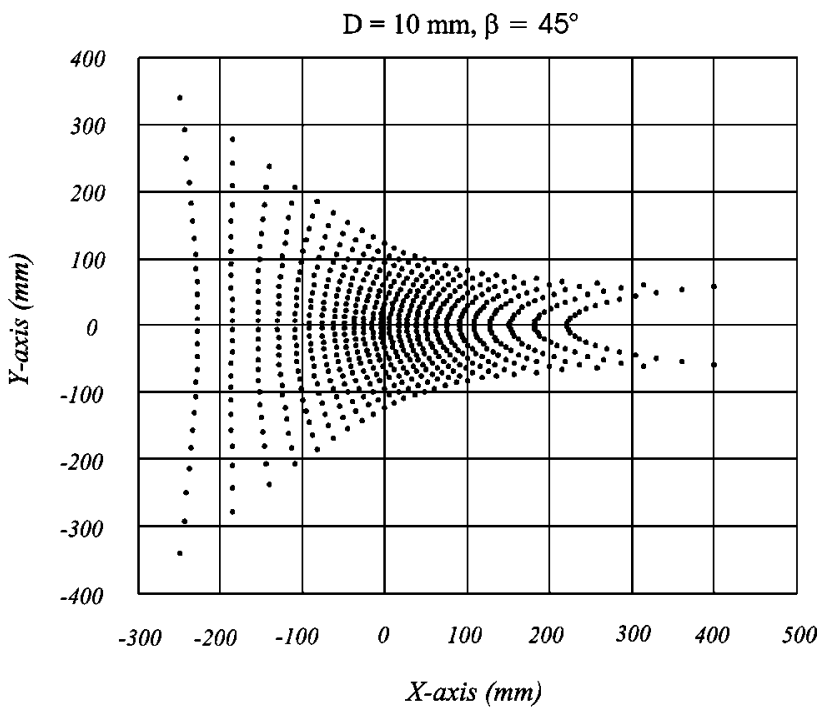

(b)

Fig. 10 (a) $X Y$ distortions on a target plane $(d=120 \mathrm{~mm})$ for tilt angles of $\left(\alpha_{X}=\alpha_{y}= \pm 30 \mathrm{deg}\right)$ when the center of rotation is at the center of reflection $(D=0)$. (b) $X Y$ distortions when $D=10 \mathrm{~mm}$ and $\beta=45$ deg. 


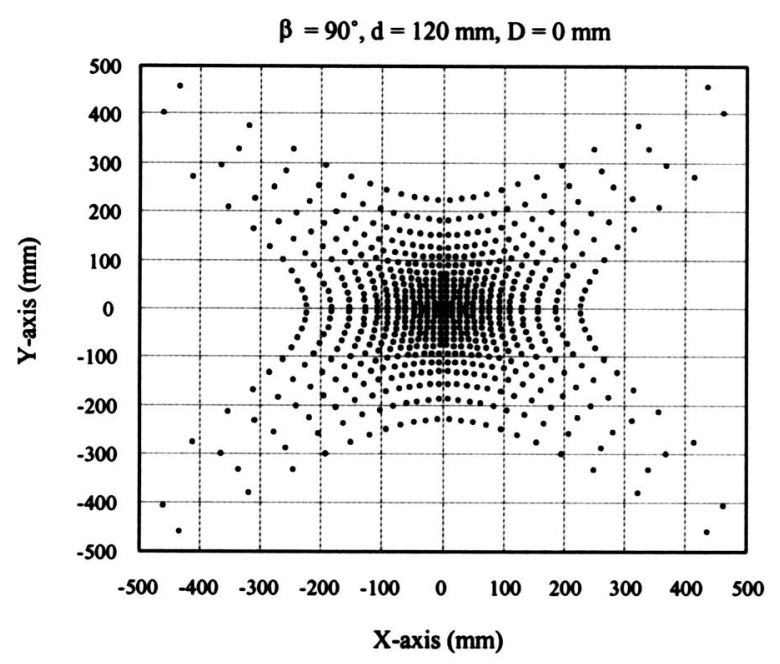

(a)

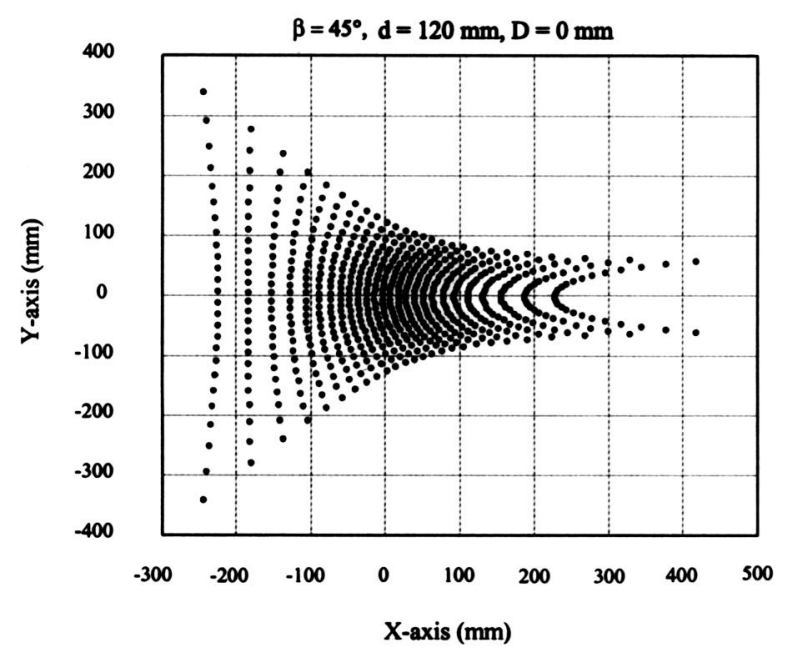

(c)

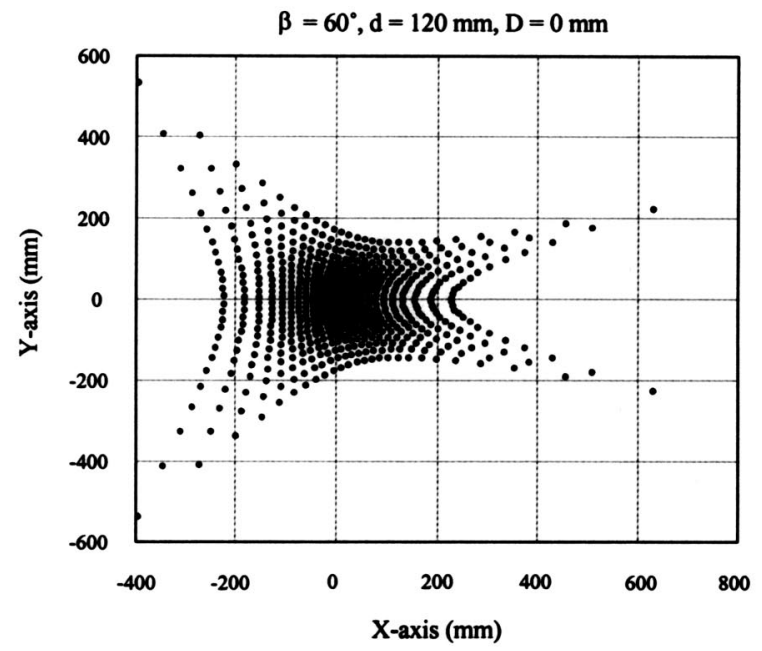

(b)

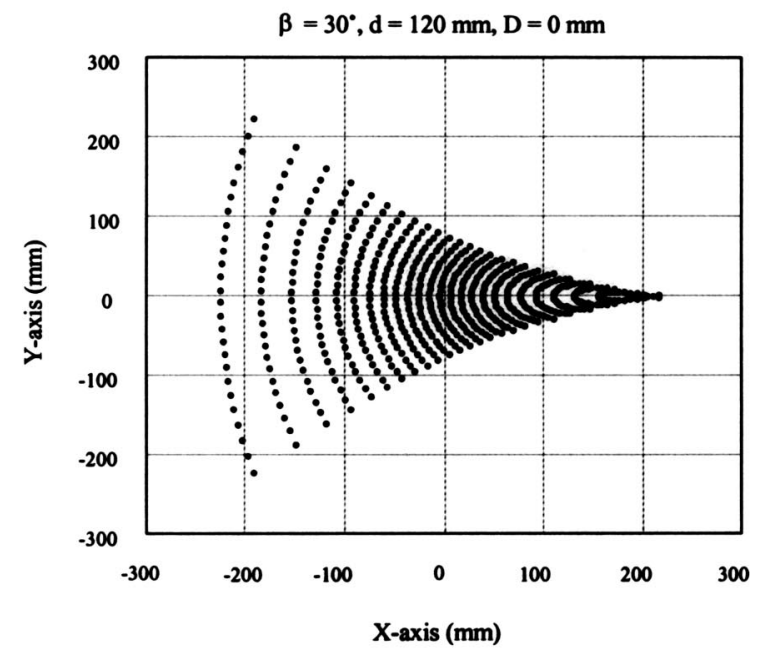

(d)

Fig. 11 (a) Distortions when the incident beam is normal to the plane of the mirror $(\beta=90$ deg, $d$ $=120 \mathrm{~mm}$, and $D=0$ ). Distortions when (b) $\beta=60 \mathrm{deg}$, (c) $\beta=45 \mathrm{deg}$, and (d) $\beta=30 \mathrm{deg}$.

$= \pm 30 \mathrm{deg}$, and a variation of $\Delta \alpha_{x}= \pm 3 \mathrm{deg}$ around $\alpha_{x}$ $=28.5 \mathrm{deg}$ for the case where $\beta_{0}$, which is the initial value of $\beta$ equal to $45 \mathrm{deg}$. The curvature changes gradually until the angle $\beta=\beta_{0}+28.5 \mathrm{deg}=73.5 \mathrm{deg}$. However, Fig. 8(b), which shows the distortion profile for the same range of $\alpha_{y}$ and a smaller deviation of $\Delta \alpha_{x}= \pm 0.5 \mathrm{deg}$ around $\alpha_{x}$ $=28.5 \mathrm{deg}$, indicates a more complex evolution of the curvature. The curves on the left-hand side are of the fourth order, whereas the curves on the right-hand side have a dominant second order coefficient. It can be noted that in a small area in-between, a linear distribution can be obtained for small tilt angles. The distortion profile for this linear region is presented in Fig. 9(a). It corresponds to scan angles of $\alpha_{y}= \pm 3 \mathrm{deg}$, and a variation of $\Delta \alpha_{x}= \pm 3 \mathrm{deg}$ around $\alpha_{x}=28.5 \mathrm{deg}$. The grid indicated in the figure corresponds to the ideal linear relation between the displacement on the target field and the scan angles $(X=Y$ $\left.=2 d \alpha_{x}=2 d \alpha_{y}\right)$. It is obvious that the errors $\Delta x$ and $\Delta y$ at this location are much more significant, as the distance between two successive lines of the grid corresponds to a theoretical shift of $0.5 \mathrm{deg}$, whereas the distance between two consecutive points represents the real shift of $0.5 \mathrm{deg}$. It can be seen that the curvature in both axes is minimal in this case. An error $\Delta x$ as small as $1 \mu \mathrm{m}$ on a surface greater than $30 \times 80 \mathrm{~mm}$ is obtained when $\alpha_{y}=3 \mathrm{deg}$ and $\alpha_{x}$ $=28.5 \mathrm{deg}$ compared to the point when $\alpha_{y}=0 \mathrm{deg}$ and $\alpha_{x}$ $=28.5 \mathrm{deg}$. This means that the two scanned lines along the $X$ and $Y$ axes, at these specific angles, are quasiorthogonal to each other. However, the change in slope of the lines [defined by the points indicated in Fig. 9(a)] on both sides of the $X$ axis is still significant. Therefore, the rectangular zone indicated is zoomed and the result is illustrated in Fig. 9(b) for scan angles of $\alpha_{y}= \pm 0.9 \mathrm{deg}$, and a variation of $\Delta \alpha_{x}= \pm 0.4 \mathrm{deg}$ around $\beta=73.5 \mathrm{deg}$. It is clear that the distortions in this area are considerably reduced. In fact, straight lines can be drawn to join the different points of the distortion profile. Despite the almost ideal behavior in this zone, it is not attractive for laser material processing applications in general, as the laser beam will irradiate the work piece with a notable inclination, leading to spots with an 
Hafez, Sidler, and Salathé: Study of the beam path distortion profiles ...

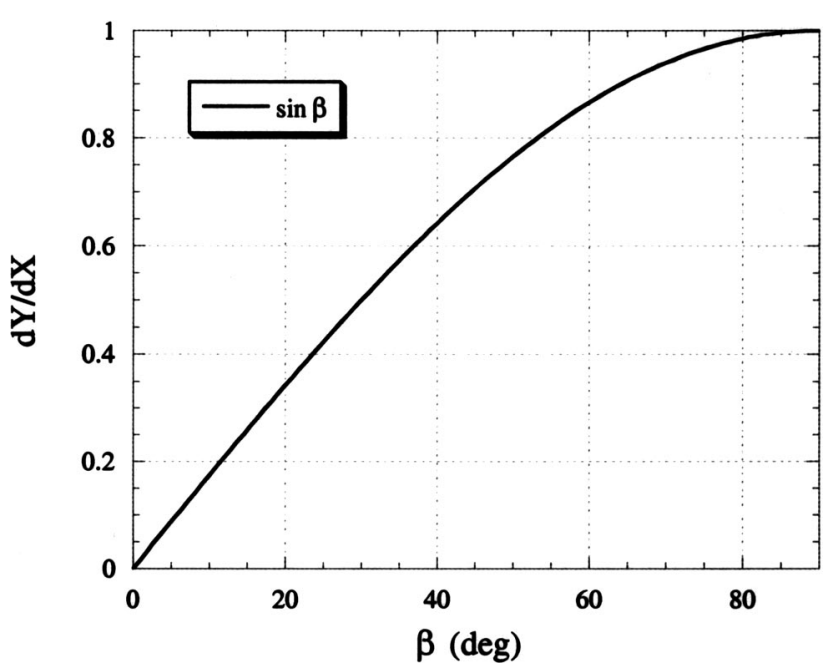

Fig. 12 The rate of change of the two amplitudes $(d Y / d X)$ as a function of the angle $\beta$.

elliptical shape. On the other hand, the linear area can be very interesting for image scanning applications and metrology based on radar and laser radar technology.

\subsection{Effect of the Optical and Geometrical Parameters on the BDP}

Several parameters are studied and their amplitudes varied to determine their influence on the nonlinear distortion profile. The following simulations are carried out for large deflections ( $\left.\alpha_{x}=\alpha_{y}= \pm 30 \mathrm{deg}\right)$ to better illustrate the distortion profile variations. Figures 10(a) and 10(b) show the distortions on a target plane located at $d=120 \mathrm{~mm}$, and for $D=0$ and $D=10$, respectively. The two profiles are almost identical. However, a slight shift toward the left can be noticed in the second case. This implies that $D$ is not the dominant parameter responsible for the distortion distribution. On the other hand, the angle $\beta$ of the laser beam has a significant effect on the distortion, as shown in Figs. 11(a) to $11(\mathrm{~d})$. In these simulations the distance $D$ is set at zero. For the extreme case where the incident beam is normal to
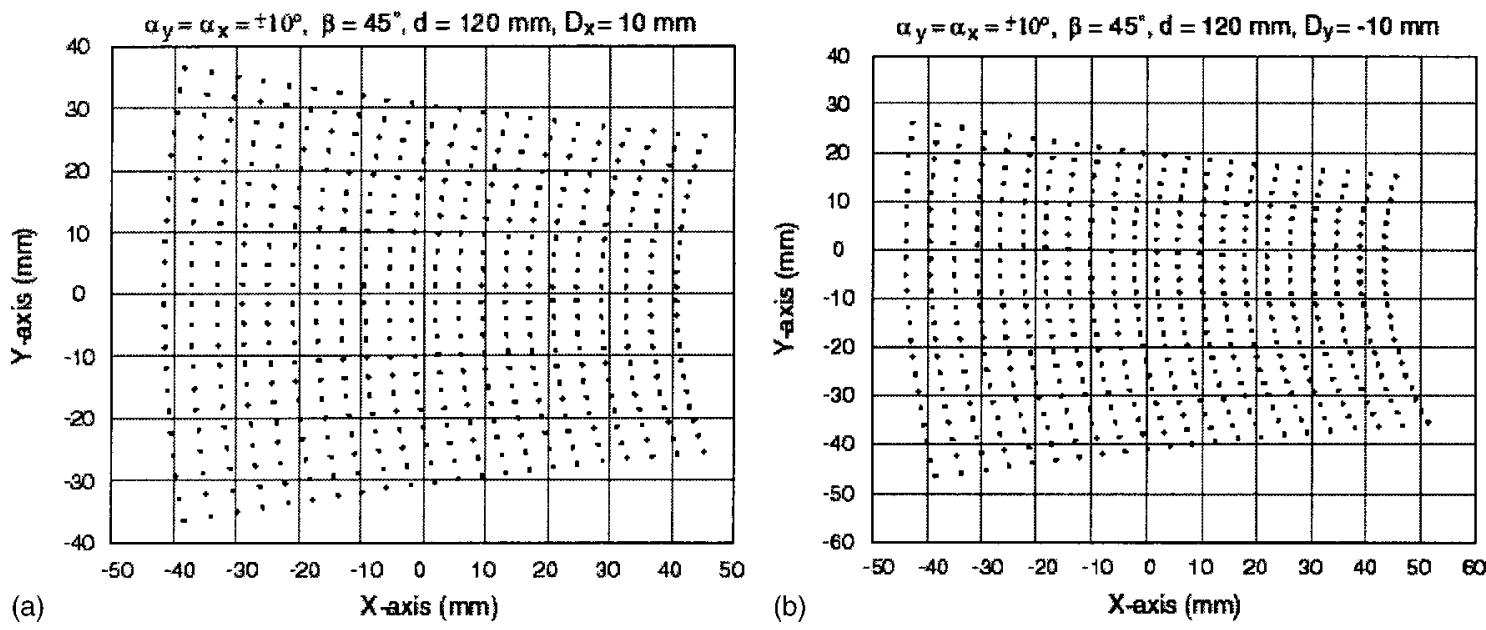

Fig. 13 Distortions profile when a shift (a) $D_{x}=10 \mathrm{~mm}$ and (b) $D_{y}=-10 \mathrm{~mm}$ is applied to the incident laser beam.

the plane of the mirror $(\beta=90 \mathrm{deg})$, the distortion reached on the target surface is of the pincushion distortion type. The pincushion effect is symmetrical around both the $X$ and $Y$ axes and has the same value [see Fig. 11(a)]. When $\beta$ is decreased $(\beta=60 \mathrm{deg})$, there is still a symmetry around the $X$ axis, whereas the distribution around the $Y$ axis is asymmetrical. Despite the different angles $\beta$, the magnitude of the scanned line on the $X$ axis $\left(\alpha_{y}=0 \mathrm{deg}\right)$ remains almost constant (from -225 to $+225 \mathrm{~mm}$ ) independently of the value of $\beta$ [see Figs. 11(a) to 11(d)]. Furthermore, it is worth noting that the displacement along $Y$ increases with the angle $\beta$. Figure 12 indicates the rate of change of the two displacements $(d Y / d X)$ when $\beta$ is increased.

Therefore, the following relation applies:

$\frac{d Y}{d X}=\sin \beta$

Another parameter that influences the distortion profile is the position of the point of impact of the incident laser beam on the mirror. A shift of the laser beam $D_{x}$ in the $X$ direction as shown in Fig. 13(a) (in this case, the tilt angles are $\alpha_{x}=\alpha_{y}= \pm 10 \mathrm{deg}$ ) will have the same effect as a shift in the center of rotation $D$ [Fig. 10(b)]. The whole profile is shifted from the $Y$ axis but the symmetry around the $X$ axis remains unchanged. Figure 13(b) shows the distortion profile when a shift $D_{y}$ is applied to the laser beam. The distribution is no longer symmetrical around the $X$ axis and the curvature along the $Y$ axis is distorted.

\subsection{Correction Factors}

To obtain a linear system in which the spot displacement on the target plane is linearly proportional to the tilt angle, the errors $\Delta x$ and $\Delta y$ must be determined for any point on the scan field. Therefore, two analytical relations for determining the correction factors as a function of the tilt angles are derived. The errors studied in this section are valid for the system presented in Fig. 2 used for microspot welding applications. In this case: $d=120 \mathrm{~mm}, D=0, \beta=45 \mathrm{deg}$, and the scan angles are varied between $\alpha_{x}=\alpha_{y}= \pm 3 \mathrm{deg}$. 


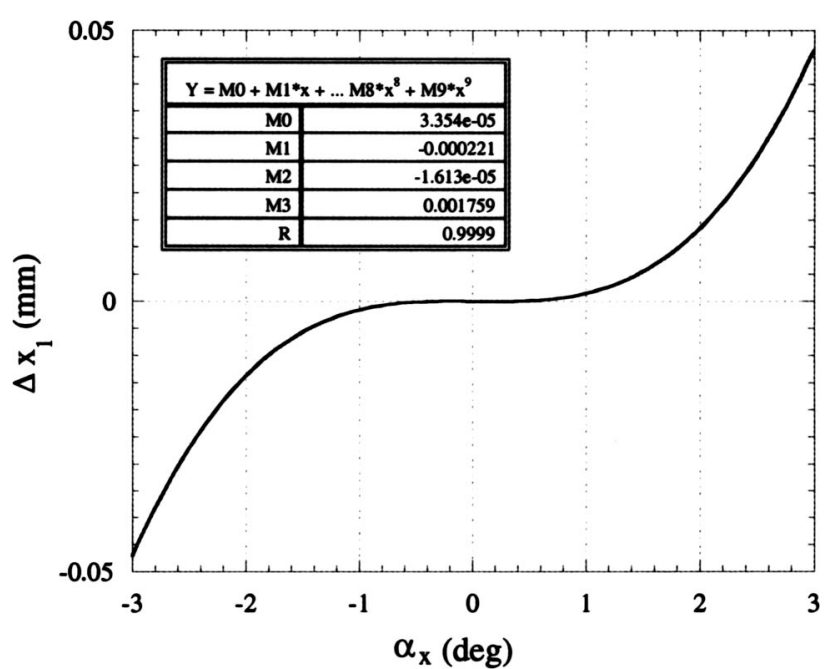

Fig. $14 \Delta x_{1}$ as a function of the tilt angle $\alpha_{x}$.

$\Delta x$ is investigated first and can be described as a function of the actual position $Y$ and the tilt angle $\alpha_{x}$ :

$\Delta x=f\left(Y, \alpha_{x}\right)=A\left(\alpha_{x}\right)+B\left(\alpha_{x}\right) \cdot Y+C\left(\alpha_{x}\right) \cdot Y^{2}$,

where $A\left(\alpha_{x}\right)$ is almost negligible for small angles $(\alpha \cong$ $\tan \alpha)$ but increases exponentially with larger angles. $A\left(\alpha_{x}\right)$ is defined as $\Delta x_{1}$. The function $B\left(\alpha_{x}\right)$ is equal to zero for the $Y$-axis nonlinearity, as the slopes are equal to zero at the minimum points of the parabolas. The function $C\left(\alpha_{x}\right)$ is multiplied by the quadratic term $Y^{2}$ and is defined as $\Delta x_{2}$. Equation (12) is rewritten as:

$\Delta x=\Delta x_{1}+\Delta x_{2}$.

$\Delta x_{1}$ is represented as a function of $\alpha_{x}$ in Fig. 14, and satisfies the following relation:

$\Delta x_{1}=M_{0}+M_{1} \alpha_{x}+M_{2} \alpha_{x}^{2}+M_{3} \alpha_{x}^{3}$.
By applying a curve fitting between the different values (see the values indicated on the different curves), it appears that the cubic coefficient is the dominant coefficient, whereas the other coefficients can be neglected. Equation (14) is rewritten as:

$\Delta x_{1}=M_{3} \alpha_{x}^{3}$.

Figure 15(a) indicates the curvature of the scanned lines for different angles $\alpha_{x}$ varying from +3 to $-3 \mathrm{deg}$. The change of curvature $C\left(\alpha_{x}\right)$ as a function of the tilt angle $\alpha_{x}$ is represented in Fig. 15(b) and satisfies the following relation:

$C\left(\alpha_{x}\right)=N_{0}+N_{1} \alpha_{x}+N_{2} \alpha_{x}^{2}+N_{3} \alpha_{x}^{3}+N_{4} \alpha_{x}^{4}$.

In this case only the cubic term can be neglected. Therefore $\Delta x_{2}$ is expressed as:

$\Delta x_{2}=\left(N_{0}+N_{1} \alpha_{x}+N_{2} \alpha_{x}^{2}+N_{4} \alpha_{x}^{4}\right) \cdot Y^{2}$.

The error $\Delta x$ from Eq. (12) is therefore expressed as:

$\Delta x=M_{3} \alpha_{x}^{3}+\left[\left(N_{0}+N_{1} \alpha_{x}+N_{2} \alpha_{x}^{2}+N_{4} \alpha_{x}^{4}\right) \cdot Y^{2}\right]$

On the other hand, the error $\Delta y$ is a function of the actual position $X$ and the tilt angle $\alpha_{y}$. Therefore, it can be written as:

$$
\Delta y=f\left(X, \alpha_{y}\right)=A^{\prime}\left(\alpha_{y}\right)+B^{\prime}\left(\alpha_{y}\right) \cdot X+C^{\prime}\left(\alpha_{y}\right) \cdot X^{2} .
$$

It should be noted from the distortion profile shown in Fig. 16(a) that the two functions $A^{\prime}\left(\alpha_{y}\right)$, which determines the shift of the curve in the $Y$ direction, and $B^{\prime}\left(\alpha_{y}\right)$, which gives the slope of the curve, have the greatest contributions in the previous relation for small angles $( \pm 3 \mathrm{deg})$. How-

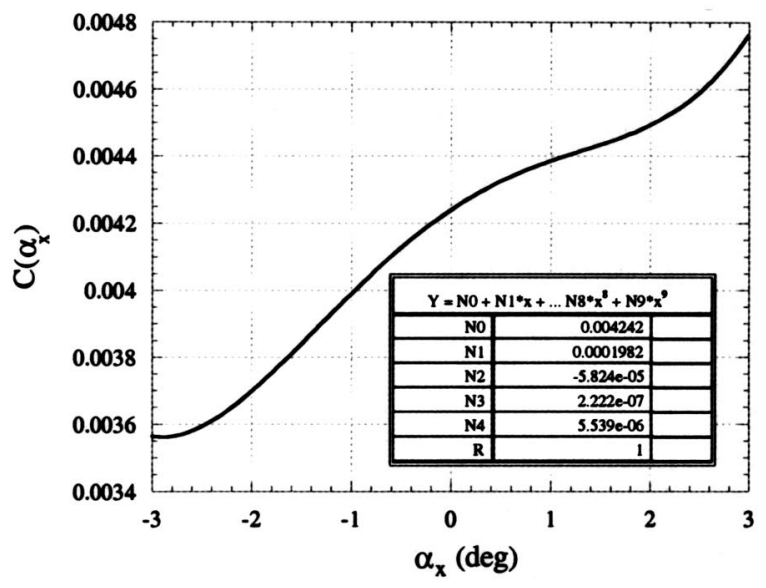

(b)

Fig. 15 (a) $\Delta x_{2}$ as a function of the distance $Y$ for different $\alpha_{x}$. (b) The change in curvature $C\left(\alpha_{x}\right)$ as a function of $\alpha_{x}$. 


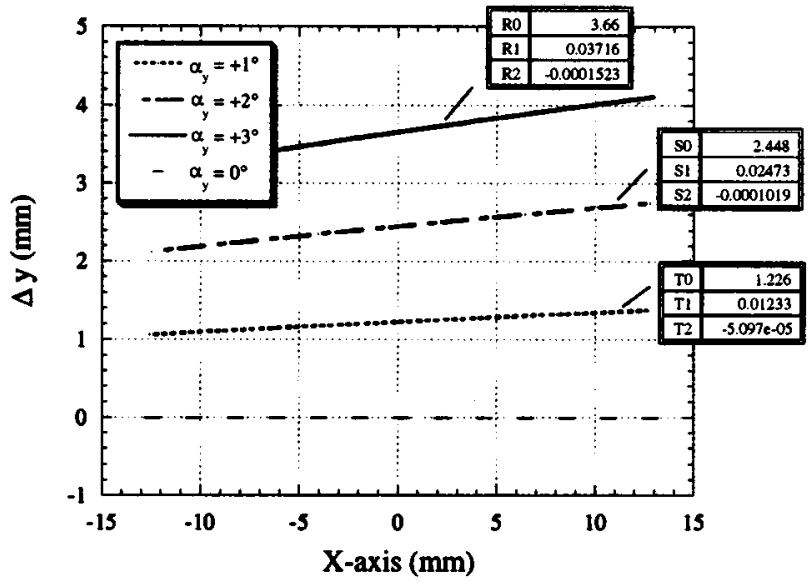

(a)

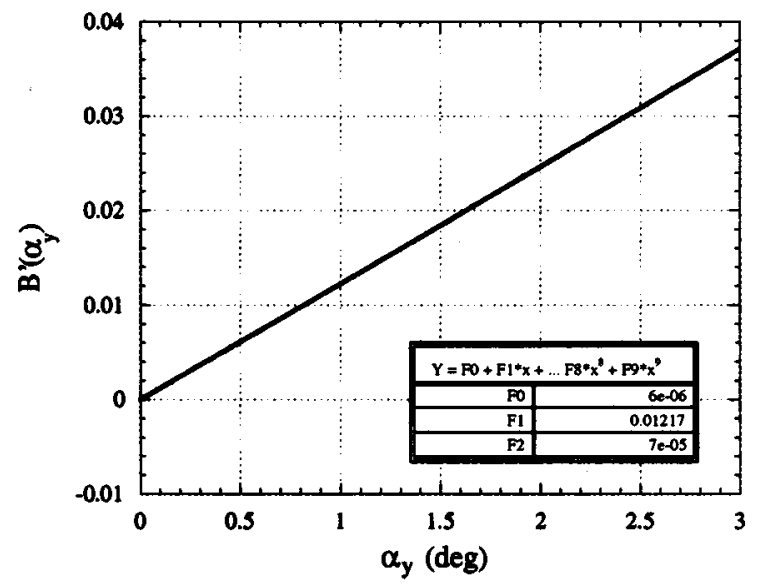

(c)

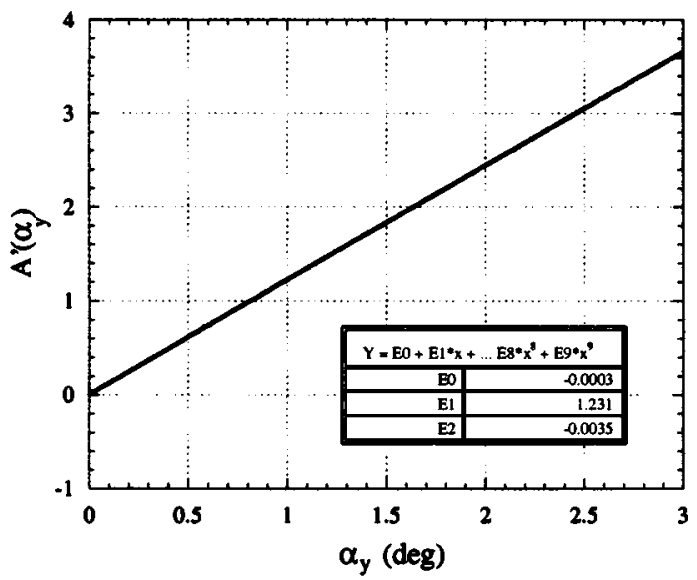

(b)

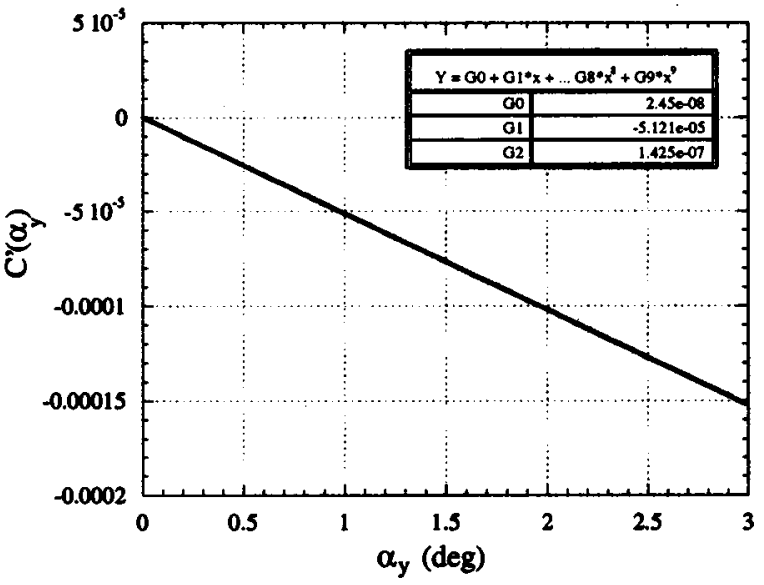

(d)

Fig. 16 (a) $\Delta y$ as a function of the distance $X$ for different values of $\alpha_{y}$. (b) $A^{\prime}\left(\alpha_{y}\right)$ as a function of $\alpha_{y}$. (c) $B^{\prime}\left(\alpha_{y}\right)$ as a function of $\alpha_{y}$. (d) $C^{\prime}\left(\alpha_{y}\right)$ as a function of $\alpha_{y}$.

ever, the quadratic term $C^{\prime}\left(\alpha_{y}\right)$ should not be neglected even for such small angles. Each of these three functions has a quadratic relation with $\alpha_{y}$ as indicated in Figs. 16(b) to $16(\mathrm{~d})$, and the three relations can be written as

$$
\begin{aligned}
& A^{\prime}\left(\alpha_{y}\right)=E_{0}+E_{1} \alpha_{y}+E_{2} \alpha_{y}^{2}, \\
& B^{\prime}\left(\alpha_{y}\right)=F_{0}+F_{1} \alpha_{y}+F_{2} \alpha_{y}^{2}, \\
& C^{\prime}\left(\alpha_{y}\right)=G_{0}+G_{1} \alpha_{y}+G_{2} \alpha_{y}^{2} .
\end{aligned}
$$

The following coefficients $E_{0}, F_{0}, G_{0}$, and $G_{2}$ can be neglected. Therefore, the error $\Delta y$ is expressed as

$$
\begin{aligned}
\Delta y= & {\left[E_{1} \alpha_{y}+E_{2} \alpha_{y}^{2}\right]+\left(\left[F_{1} \alpha_{y}+F_{2} \alpha_{y}^{2}\right] \cdot X\right) } \\
& +\left(\left[G_{1} \alpha_{y}\right] \cdot X^{2}\right) .
\end{aligned}
$$

Equations (18) and (23) lead to an accuracy level better than $0.005 \%$ for displacement along the $Y$ axis, and $0.044 \%$ for the displacements along the $X$ axis. Based on the previous vector analysis, a simplified model of the first order was derived to compensate for the scanner nonlinearity. ${ }^{10}$
The results obtained with this model were satisfactory and allowed the tracking of a microgripper by means of a CCD camera.

\section{Summary and Conclusion}

Any beam scanning system will undergo path distortions of the laser beam, whether it is a single mirror with a singlerotation DOF, two-rotation DOF, or a two-mirror configuration. In this work, the study of the distortion profiles resulting from a tip/tilt mirror is investigated in detail. A vector analysis is carried out and the main parameters that generate these distortions are identified. The angle of incidence of the laser beam is the most dominant parameter, however the effect of the distance $D$ between the center of rotation and the reflecting surface is also noticeable but much less important. Furthermore, mathematical relations for the correction factors are developed to compensate for the beam path distortions. The case of a scanning head using this scanner for microspot welding is studied. After the correction, an accuracy better than $0.005 \%$ for the $Y$ axis and $0.044 \%$ for the $X$ axis is obtained all over a target plane of $25 \times 25 \mathrm{~mm}$ located at a distance $d=120 \mathrm{~mm}$ from 
Hafez, Sidler, and Salathé: Study of the beam path distortion profiles . . .

the center of rotation of the mirror. The same procedure presented here can apply to any other case where the optical and geometrical parameters differ.

\section{Acknowledgments}

This work has been carried out in the framework of a European project on Accurate Manipulation Using Laser Technology (AMULET) sponsored by the European Community and the Swiss Government (OFES). The authors would like to thank Dr. R. de Boer from Philips CFT for the interesting and constructive discussions.

\section{References}

1. J. I. Montagu, "Galvanometric and resonant low-inertia scanners," in Optical Scanning, G. F. Marshall, Ed., vol. 31, pp. 525-613, Marcel Dekker, Inc., New York (1991).

2. K. Schönenberger, "Eye-guided laser surgery," Ph.D. thesis, Swiss Federal Institute of Technology, Lausanne (1996).

3. S. Hattori, N. Wakita, and M. Okuda, "Light deflection apparatus," U.S. patent no. 4,660,941 (April 1987).

4. L. Zago, P. Genequand, and I. Kjelberg, "Advanced flexure structures in active high-accuracy and large bandwidth mechanisms," Space Microdynamics and Accurate Control Symposium Proceedings, Centre National d'Etudes Spatiales (CNES), Toulouse (May 1997).

5. L. Masotti, "Device and method for deflecting a laser beam by means of a single mirror," European patent no. EP 0790512 A1 (Aug. 1997).

6. R. J. Pryputniewicz, "Quantitative determination of displacements and strains from holograms," chap. 3, pp. 33-74, Optical Sciences, vol. 68, Holographic Interferometry, P. K. Rastogi, Ed., SpringerVerlag, Berlin (1994).

7. P. K. Rastogi, "Techniques to measure displacements derivatives and surfaces shapes. Extension to comparative holography," chap. 7, pp. 213-292, Optical Sciences, vol. 68, Holographic Interferometry, Springer-Verlag, Berlin (1994)

8. M. Hafez, T. C. Sidler, R. P. Salathé, G. L. M. Jansen, and J. C. Compter, "Design, simulations and experimental investigations of a compact single mirror tip/tilt laser scanner," Mechatronics 10(7), 741-760 (Oct. 2000)

9. M. Hafez, K. Julliard, S. Grossmann, L. Olivetta, T. C. Sidler, R. P. Salathé, H. P. Schwob, T. Blom, and W. Hoving "A compact, multisensor laser scanning head for processing and monitoring micro-spot welding," Laser Precision Microfabrication 2000, Omiya, Japan (June 2000).

10. G. Froidevaux, "Commande par laser d'un micro-actionneur en alliage à memoire de forme," Master thesis, Swiss Federal Institute of Technology (2000)

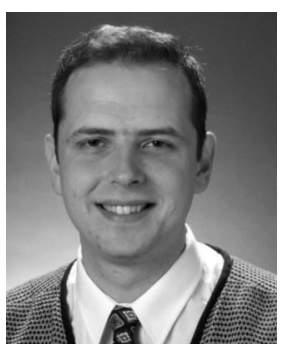

Moustapha Hafez received the degree of Bachelor of Science in mechanical engineering from the American University in Cairo in 1993. He then obtained his Master of Science in robotics from l'ENSAM in Paris in 1995 and his PhD from the Institute of Applied Optics at the Swiss Federal Institute of Technology in Lausanne, Switzerland, in 2000. During his PhD research, he designed and developed a highly innovative fast-steering compact laser scanner for high power material processing applications. He was a postdoc- toral researcher at the Massachusetts Institute of Technology (MIT) in the Field and Space Robotics Laboratory in 2001, where he was the lead researcher of the NASA Institute for Advanced Concepts project on self-transforming robotic planetary explorers. He is now the head of the mechatronics group at the Atomic Energy Commission in France at Fontenay aux Roses. His research interests lie in the field of mechatronics, optical scanning, dynamics, robotics, microengineering, and smart materials.

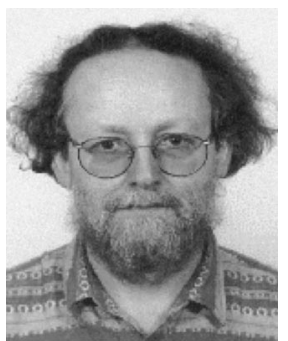

Thomas Sidler did his studies in physics at the University of Neuchatel from 1965 to 1971. During the following five years, he worked as a research assistant at the Physics Institute of the University of Neuchatel, in the group of solid state physics with Professor J. Rossel, finishing his PhD in 1976 with the subject "Vk-centers in cesium iodide." From 1976 to 1982, he continued with his research activities at the $\mathrm{Mi}$ crotechnics Institute in different domains: fiber optics, holographic interferometry, optical pattern recognition, and signal processing. From 1982 to 1986, he was a research and research group manager at Asulab, central laboratories of the Societe de Microelectronique d'Horlogerie, essentially working on high stability $\mathrm{CO}_{2}$ lasers for metrology and high power, high beam quality Nd-YAG lasers for precision material processing. Since 1992, he has been doing research on laser source development and laser applications in material processing at The Swiss Federal Institute of Technology.

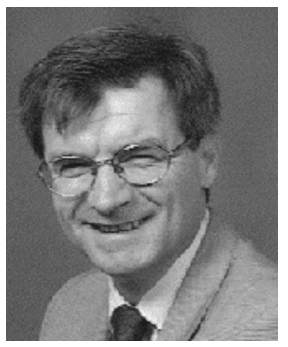

René-Paul Salathé is a professor of applied optics at the Swiss Federal Institute of Technology in Lausanne (EPFL), Switzerland. He received the MS, PhD, and Habilitation (Privatdozent) degrees in 1970 1974, and 1979, respectively, from the University of Bern, where he was actively engaged in research on semiconductor lasers. During 1977 to 1978 he spent a year at Bell Telephone Laboratories, Murray Hill, New Jersey, working in the field of integrated optics. From 1978 to 1983 he directed a research group engaged on laser processing at the University of Bern. In 1984, he became head of the section "Technology," and in 1985, head of the division "Material Testing and Technology" at the research and development center of the Swiss PTT. From 1989 to 2001 he was directing the Institute of Applied Optics in the Microengineering Department of EPFL. From 1995 to 1998 he was head of the microengineering department. During the winter semester 1998 to 1999 he was on a sabbatical leave at the Center for Biological and Medical Engineering at the University of Texas in Austin. Since 2002 he has been heading the Institute "Imagerie et Optique Appliquée" (IOA) within the Faculty of Engineering Sciences and Techniques at EPFL. 